\title{
Estimation of Weak Lensing Parameters by Stochastic Integration
}

\section{Report}

Author(s):

Petersen, Wesley P.

Publication date:

2000-06

Permanent link:

https://doi.org/10.3929/ethz-a-004289446

Rights / license:

In Copyright - Non-Commercial Use Permitted

Originally published in:

SAM Research Report 2000-06 


\title{
Estimation of Weak Lensing Parameters by Stochastic Integration
}

\author{
W.P. Petersen
}

Research Report No. 2000-06

June 2000

Seminar für Angewandte Mathematik

Eidgenössische Technische Hochschule

CH-8092 Zürich

Switzerland 


\title{
Estimation of Weak Lensing Parameters by Stochastic Integration
}

\author{
W.P. Petersen \\ Seminar für Angewandte Mathematik \\ Eidgenössische Technische Hochschule \\ CH-8092 Zürich \\ Switzerland
}

Research Report No. 2000-06 June 2000

\begin{abstract}
A procedure for computing distance-redshift statistics in inhomogeneous universes is described. Interpreting the generalized Dyer-Roeder equation as a stochastic differential equation permits a treatment of the shearing forces as Brownian movement and yields a straightforward Monte-Carlo simulation. Shear distributions taken from N-body simulations give a redshift dependent diffusion coefficient which is used to produce a $C^{0}$ analytical model approximating the Weyl term in Sachs' equations.
\end{abstract}

Keywords: cosmology: theory, gravitational lensing, distance scale, methods: numerical, statistical 


\section{Introduction}

Computing curvature of geodesics and shear of nearby light rays due to inhomogeneous sources of matter is a long standing problem. The expansion of matter on large scales can be more than $3000 \mathrm{~km} / \mathrm{sec}$. at the current epoch, whereas the light propagation velocity is $c, 100$ times faster. To resolve effects out to roughly the observable horizon, say $z \sim 5$, of inhomogeneities whose mass is less than galaxy size would then require simulations of volumes whose sides are order of $5 \mathrm{Gpc}$., which is infeasible with current computer technology. In the following we ask for a simpler result: if a simulation can resolve inhomogeneities at roughly a galaxy mass scale, can we take a representative volume whose size is still much larger than a cluster correlation length and use the statistics of the shearing force in this volume to model increments for the evolution of shear $\sigma$ at a larger scale? For example, the simplest physically reasonable model for the evolution of $\sigma$ should be a Markov process continuous in the redshift $z$.

We start with the generalized Dyer-Roeder $(1972,1973)$ equation for the angular diameter distance $D(z)$ as a function of redshift $z$. Our principle idea is to treat $\sigma(z)$ and possibly smoothness, $\tilde{\alpha}(z)$, as continuous $\left(C^{0}\right)$ stochastic parameters. For in that case, $D(z)$ has well defined and continuous second derivatives:

$$
(1+z)(1+\Omega z) \frac{d^{2} D}{d z^{2}}+\left(\frac{7}{2} \Omega z+\frac{\Omega}{2}+3\right) \frac{d D}{d z}+\left(\frac{3}{2} \tilde{\alpha}(z) \Omega+\frac{|\sigma(z)|^{2}}{(1+z)^{5}}\right) D=0 .
$$

The shear component is computed by integrating over the Weyl term, $\mathcal{F}$, in Sachs' equations (see Schneider, Ehlers and Falco (hereafter SEF), section 3.4.2, 1992). We argue that at any length much larger than a typical cluster scale, the parameter $\mathcal{F}$ can be approximated by a Gaussian model whose real and imaginary parts can be treated independently. The variance of a unitless form decreases roughly like $a^{2}$, where $a$ is the Friedmann expansion factor. Large scale behavior then has a shearing force which behaves like Brownian motion having a variable diffusion coefficient (in $z$ ).

We write the shear as in SEF,

$$
\sigma(z)=-\frac{1}{2} \int_{0}^{z}\left(\frac{D(\xi)}{D(z)}\right)^{2}(1+\xi)^{-3}(1+\Omega \xi)^{-\frac{1}{2}} T(\xi) d \xi
$$

where $T$ is proportional to the Weyl term in Sachs' equations. This is

$$
\begin{aligned}
T(\xi) & =-2\left(\frac{c}{H_{0}}\right)^{2} \mathcal{F} \\
& =-2 \frac{1}{H_{0}^{2}}(1+\xi)^{2} \frac{d^{2} \Phi}{d z^{2}} .
\end{aligned}
$$

Here, $\mathbf{z}=x_{1}+i x_{2}$ is the complex 2-plane orthogonal to the local congruence of the light ray, and $\Phi\left(x_{1}, x_{2}, x_{3}\right)$ is the local gravitational potential. The real and imaginary parts of the shearing force are

$$
\Phi_{\mathrm{zz}} \equiv \frac{d^{2} \Phi}{d \mathrm{z}^{2}}=\left(\Phi_{11}-\Phi_{22}\right)-2 i \Phi_{12}
$$


Thus,

$$
\sigma(z)=-\frac{1}{H_{0}^{2}} \int_{0}^{z}\left(\frac{D(\xi)}{D(z)}\right)^{2}(1+\xi)^{-1}(1+\Omega \xi)^{-\frac{1}{2}} \Phi_{\mathrm{zz}}(\xi) d \xi .
$$

Our main point is to treat the shearing force, $\Phi_{\mathrm{zz}}$, as a continuous complex random variable in $z$ about which we can extract information from N-body simulations. It is helpful to put (6) into a unitless form. Using the mean mass density $\rho=\Omega \rho_{c}=\Omega\left(3 H_{0}^{2} /(8 \pi G)\right)$, and defining a local number density $n(\vec{x})$ in unitless coordinates, we get

$$
\sigma(z)=-\frac{3 \Omega}{8 \pi} \int_{0}^{z}\left(\frac{D(\xi)}{D(z)}\right)^{2}(1+\xi)^{2}(1+\Omega \xi)^{-\frac{1}{2}} \mathcal{R} d \xi .
$$

The function $\mathcal{R}(\xi)=\mathcal{R}^{[1]}-i \mathcal{R}^{[2]}$ is $\Phi_{\mathrm{zz}}$ in unitless 3 -D coordinates, $\vec{x}$, and whose real and (negative of) imaginary parts are

$$
\begin{aligned}
& \mathcal{R}^{[1]}=\int d^{3} y n(y)\left[\left(\frac{1}{|x-y|}\right)_{11}-\left(\frac{1}{|x-y|}\right)_{22}\right] \\
& \mathcal{R}^{[2]}=2 \int d^{3} y n(y)\left[\left(\frac{1}{|x-y|}\right)_{12}\right] .
\end{aligned}
$$

Our first objective is to find sampling statistics for these two components. We extract our statistics from an N-body simulation at each red-shift epoch and resolve the shearing forces in a cube containing $L^{3}$ cells. The samples of interest will be measured in pixels $(0 \leq a, b \leq L-1)$ in the 2-dimensional plane orthogonal to the ray direction (say $\left.x_{3}\right)$. Box-size, $L$, was chosen to large enough to be able to neglect the tails (we picked $L=128$ ) to an accuracy of the N-body simulation that fills the $L^{3}$ cells. Mean square errors in force calculations of the adaptive $P^{3} M$ code, Couchman (1991), are less than 2 percent. In the lattice of cells, $\mathcal{R}$ has the components

$$
\begin{aligned}
& \mathcal{R}_{a, b, c}^{[1]}=\sum_{p=0}^{L-1} \sum_{q=0}^{L-1} \sum_{r=0}^{L-1} N_{(a, p),(b, q),(c, r)}^{[1]} \frac{p^{2}-q^{2}}{\left(p^{2}+q^{2}+r^{2}+\epsilon^{2}\right)^{\frac{5}{2}}} \\
& \mathcal{R}_{a, b, c}^{[2]}=\sum_{p=0}^{L-1} \sum_{q=0}^{L-1} \sum_{r=0}^{L-1} N_{(a, p),(b, q),(c, r)}^{[2]} \frac{2 p q}{\left(p^{2}+q^{2}+r^{2}+\epsilon^{2}\right)^{\frac{5}{2}}},
\end{aligned}
$$

and $\epsilon$ is a softening parameter. The appropriately symmetrized number densities covering each octant are, each index modulo $L$ due to the periodic boundary conditions,

$$
\begin{array}{r}
N_{(a, p),(b, q),(c, r)}^{[1]}=\frac{1}{\langle n\rangle}\left(\begin{array}{c}
n_{a+p, b+q, c+r}+n_{a-p, b+q, c+r}+n_{a+p, b-q, c+r}+n_{a+p, b+q, c-r}+ \\
n_{a-p, b-q, c+r}+n_{a-p, b+q, c-r}+n_{a+p, b-q, c-r}+n_{a-p, b-q, c-r}
\end{array}\right) \\
N_{(a, p),(b, q),(c, r)}^{[2]}=\frac{1}{\langle n\rangle}\left(\begin{array}{c}
n_{a+p, b+q, c+r}-n_{a-p, b+q, c+r}-n_{a+p, b-q, c+r}+n_{a+p, b+q, c-r}+ \\
n_{a-p, b-q, c+r}-n_{a-p, b+q, c-r}-n_{a+p, b-q, c-r}+n_{a-p, b-q, c-r}
\end{array}\right) .
\end{array}
$$

The mean number density per lattice volume $\langle n\rangle$ was unity in our simulations. In unitless coordinates $\langle n\rangle=L^{-3} \sum_{a, b, c=0}^{L-1} n_{a, b, c}=1$. 


\section{Choice of increments}

We wish to construct a model (say $\mathcal{Q}$ ) for $\mathcal{R}(\xi)$. But first some notation: column means, $\langle.\rangle_{\|}$, are defined by

$$
\langle Z\rangle_{\|}\left(\vec{x}_{2}\right)=\frac{1}{\Delta x_{3}} \int_{x_{3}-\Delta x_{3} / 2}^{x_{3}+\Delta x_{3} / 2} d x_{3} Z\left(\vec{x}_{2}, x_{3}\right)
$$

for any quantity $Z(x)$. In the discrete case, where $0 \leq p, q, \leq L-1$ number the $x_{1}, x_{2}$ pixels of the two dimensional sample, this is

$$
\left(\langle Z\rangle_{\|}\right)_{p, q}=\frac{1}{L} \sum_{r=0}^{L-1} Z_{p, q, r}
$$

Within this plane, orthogonal to the ray direction $x_{3}$, the sample means are defined by

$$
\langle Y\rangle_{\perp}=\frac{1}{L^{2}} \sum_{p, q=0}^{L-1} Y_{p, q}
$$

for some random variable $Y$ whose domain is in this plane. If the universe is on-average homogeneous and isotropic, we should have an expectation value for our model

$$
\langle\mathcal{Q}(\xi)\rangle_{\perp}=0
$$

Furthermore, the distribution of $\mathcal{Q}$ should be symmetric about this mean. Two choices are evident with respect to relatively small changes $\Delta z=z_{i}-z_{i-1}$ at redshift $z_{i}$. The first is by deflection planes,

2-D projection model (for example, Blandford and Narayan (1986), Schneider and Weiss (1986a, 1986b), and Jain et al. (1999))

$$
\mathcal{Q}=\frac{4}{3} \Delta z \delta\left(\xi-z_{i}\right) \mathcal{S}(\xi)
$$

where $\Delta z$ is the redshift depth of the representative volume at redshift $z_{i}$. Complex random variable $\mathcal{S}=\mathcal{S}^{[1]}-i \mathcal{S}^{[2]}$ is the shearing force $\left(\Psi_{\mathrm{zz}}\right.$, equations $(5)$ and $\left.(20)\right)$ in unitless coordinates of a deflecting plane where $\Psi$ is a 2-potential computed from the surface density $\Sigma$ of column sums of cell densities (20). The real and (negative of) imaginary parts are respectively,

$$
\begin{aligned}
& \mathcal{S}_{a b}^{[1]}=\frac{1}{\langle\Sigma\rangle} \sum_{p, q=0}^{L-1} \frac{p^{2}-q^{2}}{\left(p^{2}+q^{2}+\epsilon^{2}\right)^{2}}\left(\Sigma_{a+p, b+q}+\Sigma_{a+p, b-q}+\Sigma_{a-p, b+q}+\Sigma_{a-p, b-q}\right), \\
& \mathcal{S}_{a b}^{[2]}=\frac{1}{\langle\Sigma\rangle} \sum_{p, q=0}^{L-1} \frac{2 p q}{\left(p^{2}+q^{2}\right)^{2}+\epsilon^{2}}\left(\Sigma_{a+p, b+q}-\Sigma_{a+p, b-q}-\Sigma_{a-p, b+q}+\Sigma_{a-p, b-q}\right),
\end{aligned}
$$


where again, all indices are modulo $L$. In our simulations, the mean column density is $\langle\Sigma\rangle_{\perp}=L$. The surface density per $a, b$ pixel is $\Sigma_{a b}=\sum_{c=0}^{L-1} n_{a b c}$ as in (10). This is an impulse approximation wherein the shear is piecewise constant in each interval $z_{i+1}<z<z_{i}$. Hence, $D(z)$ is only $C^{1}$ continuous in that case. As we see below, for that situation in the limit of very small step-sizes $\Delta z$, if the variance of the shearing force is bounded, the shear vanishes. Our proposed model is more flexible and mathematically sound since it leads to a smoother $D(z) \in C^{2}$. It is a

\section{Continuous model for $\sigma$}

$$
\mathcal{Q}=\mathcal{A}(\xi)+\mathcal{B}(\xi) \frac{d w(\xi)}{d \xi}
$$

where $w(\xi)$ is a complex Brownian motion (e.g. see Doob (1953)). If only the $\mathcal{A}$ term were present and is sufficiently singular, this is the deflecting planes model. Random variable $\mathcal{A}$ must satisfy $\langle\mathcal{A}\rangle_{\perp}=0$ if the space is on-average isotropic and homogeneous. For the same reason, $\mathcal{B}$ must be stochastically independent of $d w(\xi)$. When $\mathcal{A}=0, \sigma$ will be a continuous Markov process. We compute $\mathcal{B}(\xi)$ in what follows. A more sophisticated theory might include such a symmetrically distributed, zero-centered random variable $\mathcal{A}$, but such an inclusion poses problems with continuity in (1).

For a large fixed step-size, $\Delta z$, these two models agree if the localized mass distribution approximation at $x_{3}=0$,

$$
\begin{aligned}
\Delta x_{3}\left\langle\Phi_{i j}\right\rangle_{\|} & =\int_{-\Delta x_{3} / 2}^{\Delta x_{3} / 2} \Phi_{i j}\left(\vec{x}_{2}, x_{3}\right) d x_{3} \\
& \approx \frac{c^{2}}{2} \Psi_{i j}\left(\vec{x}_{2}\right),
\end{aligned}
$$

holds, and the distributions of both are Gaussian (sampled over the $\vec{x}_{2}$ points, i.e. pixels). In the $\mathbf{z}=x_{1}+i x_{2}$ plane, $\vec{x}_{2}=\left(x_{1}, x_{2}\right)$ are the coordinates orthogonal to the congruence in direction $x_{3}$. The 2-potential is

$$
\Psi\left(\vec{x}_{2}\right)=\left(\text { constant in } \vec{x}_{2}\right)+\frac{4 G}{c^{2}} \int d^{2} y \Sigma\left(\vec{y}_{2}\right) \log \left|\vec{x}_{2}-\vec{y}_{2}\right|
$$

(e.g. Seitz and Schneider, 1994). Here, $\Sigma\left(\vec{x}_{2}\right)=\Delta x_{3}\langle\rho(\vec{x})\rangle_{\|}$is the column projected surface density, $\int_{\Delta x_{3}} \rho d x_{3}$, for a spatial depth of $\Delta x_{3}$. When $\Delta x_{3}$ is large and the mass is very localized, equation (19) is easy to show from the identity $\int_{-\infty}^{\infty} d s /\left(t^{2}+s^{2}\right)^{5 / 2}=$ $4 /\left(3 t^{4}\right)$. In a more general setting, this has been shown for properly softened potentials by Couchman et al. (1998). Here we confirm this, but only for $\epsilon \geq 3$. 


\section{Changing step-sizes}

Let us now see what happens by comparing the two models were the step-size $\Delta z$ made smaller to reduce truncation errors in computing $\sigma$. For the deflecting planes model, we make the following assumptions. Sampling in the z plane, the on-average isotropic assumption says $\langle\mathcal{S}\rangle_{\perp}=0$. Additionally, we assume

$$
\left\langle|\mathcal{S}|^{2}\right\rangle_{\perp} \leq K_{1}
$$

that is, the variance around this zero mean is bounded. This boundedness is clearly true in our simulations and is physically reasonable. Since $\langle\mathcal{S}\rangle_{\perp}=0$, then $\langle\sigma\rangle_{\perp}=0$, but the variance of $\sigma$ is

$$
\begin{aligned}
\left\langle|\sigma(z)|^{2}\right\rangle_{\perp}= & \left(\frac{3}{8 \pi}\right)^{2} \sum_{i=0}^{\lfloor z / \Delta z\rfloor} \sum_{j=0}^{\lfloor z / \Delta z\rfloor}\left(D\left(z_{i}\right) / D(z)\right)^{2}\left(D\left(z_{j}\right) / D(z)\right)^{2}\left(1+z_{i}\right)^{2}\left(1+z_{j}\right)^{2} \times \\
& \left(1+\Omega z_{i}\right)^{-\frac{1}{2}}\left(1+\Omega z_{j}\right)^{-\frac{1}{2}}(\Delta z)^{2}\left\langle\overline{\mathcal{S}}\left(z_{i}\right) \mathcal{S}\left(z_{j}\right)\right\rangle_{\perp} \\
\leq & \left(\frac{3}{8 \pi}\right)^{2}(1+z)^{4} K_{1} \sum_{i=0}^{\lfloor z / \Delta z\rfloor}(\Delta z)^{2} \\
\leq & \left(\frac{3}{8 \pi}\right)^{2}(1+z)^{4} K_{1} z \Delta z \\
\rightarrow & 0 \text { as } \Delta z \rightarrow 0 .
\end{aligned}
$$

Here we have used the independence of the increments dictated by the Markov nature of the evolution, $\left(\left\langle\overline{\mathcal{S}}\left(z_{i}\right) \mathcal{S}\left(z_{j}\right)\right\rangle_{\perp}=0\right.$, if $\left.i \neq j\right)$, the bound (21), and the $\tilde{\alpha}=0$ focusing theorem $\left|D\left(z^{\prime}\right) / D(z)\right|<1$ when $z^{\prime}<z$. A process $\sigma$ with zero mean and with vanishing absolute variance is zero. Thus $\sigma \rightarrow 0$ as the step size $\Delta z \rightarrow 0$. Whereas it is true that the step-size $\Delta z$ approaching zero loses physical meaning in the deflecting screens model, mathematically it means the numerical integration procedure is unstable: it diverges to zero as the step-size decreases. Integrating over a point process leads to inconsistent results.

To examine what happens in the continuous $\sigma$ model (18), first recall that the on-average isotropic assumption requires $\langle\mathcal{A}\rangle_{\perp}=0$. If $\mathcal{B}$ and $d w$ are independent, by equation (25) the second term will also have zero mean. For simplicity, we assume the two components of (18) are independent, $\langle\mathcal{A}(z) \mathcal{B}(z)\rangle_{\perp}=\langle\mathcal{A}(z)\rangle_{\perp}\langle\mathcal{B}(z)\rangle_{\perp}=0$, although this isn't really necessary to claim $\sigma$ does not vanish in this model. Putting bounds on the variances of these components,

$$
\begin{aligned}
\left\langle|\mathcal{A}|^{2}\right\rangle_{\perp} & \leq K_{2} \\
\left\langle|\mathcal{B}|^{2}\right\rangle_{\perp} & \leq K_{3} .
\end{aligned}
$$

Again, these bounds are physically reasonable, and for $K_{3}$ also true in simulation, where we made the smoothness simplification $\mathcal{A}=0$. A little Brownian motion calculus is 
appropriate here. The increments of a complex Brownian motion $d w=\frac{1}{\sqrt{2}}\left(d w_{1}+i d w_{2}\right)$ $\left(w_{1}\right.$ and $w_{2}$ are independent) satisfy

$$
\begin{aligned}
\langle d w(s)\rangle_{\perp} & =0 \\
\langle d \bar{w}(s) d w(t)\rangle_{\perp} & =d s d t \delta(s-t),
\end{aligned}
$$

(for example, see Kloeden and Platten (1992), Milstein (1995), Petersen (1998)). Since the increments $d w$ are Gaussian, their distribution is completely determined by these two conditions. For finite steps $\Delta z$, we have

$$
\begin{aligned}
\left\langle\Delta w\left(z_{i}\right)\right\rangle_{\perp} & =\int_{s=z_{i}}^{z_{i}+\Delta z}\langle d w(s)\rangle_{\perp}=0 \\
\left\langle\Delta \bar{w}\left(z_{i}\right) \Delta w\left(z_{j}\right)\right\rangle_{\perp} & =\int_{s=z_{i}}^{z_{i}+\Delta z} \int_{t=z_{j}}^{z_{j}+\Delta z}\langle d \bar{w}(s) d w(t)\rangle_{\perp} \\
& =\Delta z \delta_{i j} .
\end{aligned}
$$

Repeating the same sort of bound as equation (22), we get

$$
\begin{aligned}
\left\langle|\sigma(z)|^{2}\right\rangle_{\perp}= & \left(\frac{3}{8 \pi}\right)^{2} \sum_{i=0}^{\lfloor z / \Delta z\rfloor} \sum_{j=0}^{\lfloor z / \Delta z\rfloor}\left(D\left(z_{i}\right) / D(z)\right)^{2}\left(D\left(z_{j}\right) / D(z)\right)^{2}\left(1+z_{i}\right)^{2}\left(1+z_{j}\right)^{2} \times \\
& \left(1+\Omega z_{i}\right)^{-\frac{1}{2}}\left(1+\Omega z_{j}\right)^{-\frac{1}{2}} \times \\
& \left(\left\langle\overline{\mathcal{A}}\left(z_{i}\right) \mathcal{A}\left(z_{j}\right)\right\rangle_{\perp}(\Delta z)^{2}+\left\langle\overline{\mathcal{B}}\left(z_{i}\right) \mathcal{B}\left(z_{j}\right)\right\rangle_{\perp}\left\langle\Delta \bar{w}\left(z_{i}\right) \Delta w\left(z_{j}\right)\right\rangle_{\perp}\right) \\
\leq & \left(\frac{3}{8 \pi}\right)^{2}(1+z)^{4} z\left(K_{2} \Delta z+K_{3}\right)
\end{aligned}
$$

where only the first, the $K_{2}$ (the $|\mathcal{A}|^{2}$ ) term, shrinks. These are well known results for point processes, see for example Feller (1974). The conditions can be relaxed somewhat. It is not necessary that variable $\mathcal{A}$ and the B-motion $w(z)$ be independent to show the resulting $\sigma$ will not vanish as $\Delta z \rightarrow 0$. The $\lfloor z / \Delta z\rfloor$ (number of) cross terms will be each $O\left((\Delta z)^{3 / 2}\right)$, thus adding an $O\left((\Delta z)^{1 / 2}\right)$ term in the parenthesis on the right hand side of $(27)$.

We extract the $\mathcal{B}(z)$ parameter used in (18) from the variance of the column means of the shearing force

$$
\left|\mathcal{B}\left(z_{i}\right)\right|^{2}=\Delta z\left\langle\left|\langle\mathcal{R}\rangle_{\|}\right|^{2}\right\rangle_{\perp}
$$

where the phase of $\mathcal{B}$ may be arbitrarily chosen to be zero because the real and imaginary parts of $d w$ are statistically independent. We find from simulation that $\mathcal{B}(z)$ varies as a constant power (close or equal to 1) of the Friedmann expansion factor $a(z) \propto 1 /(1+z)$. See the exponents in Table 1. Explicitly, with respect to the $L^{3}$ cells of the simulation this is

$$
\left|\mathcal{B}\left(z_{i}\right)\right|^{2}=\frac{\Delta z}{L^{2}} \sum_{p, q=0}^{L-1}\left|\frac{1}{L} \sum_{r=0}^{L-1}\left[\left(\mathcal{R}^{[1]}\right)_{p, q, r}-i\left(\mathcal{R}^{[2]}\right)_{p, q, r}\right]\right|^{2}
$$


where $\mathcal{R}$ is given by $(9)$, and $z_{i}$ is the redshift epoch of the representative volume. If $\langle\mathcal{R}\rangle_{\perp}$ is equal to $\frac{4}{3} \mathcal{S}$, see equations $(16,17)$, and the distributions are Gaussian, then at large fixed step-size, we get the results, if not the philosophy, of the successive deflection planes model.

The important difference between our Brownian motion model (18) and the deflection planes model is the continuity of the shear (7) in $z$ which implies that the angular diameter distance in equation (1) makes sense: $D(z) \in C^{2}$. When the shear from equation (7) is only piecewise continuous, there is no consistent method of integrating it (1). Indeed, because the shearing force in that case is a point process, not a continuous one, in the limit of very small step sizes the shear vanishes - as we have seen in (22).

\section{$4 \quad$ N-body simulation and its statistics}

We used an adaptive particle-particle, particle-mesh $\left(A P^{3} M\right)$ code Couchman (1991) to simulate the evolution of a (128 megaparsec $)^{3}$ volume through an expansion of $1 \leq a \leq 24$, where $a$ is the Friedmann expansion factor. The relation between redshift $z$ and the Friedmann factor $a$ (where $a_{0}=24$ ) is

$$
z=\frac{a_{0}}{a}-1
$$

Beginning at $a=4(z=5)$, coordinate data for $2^{21}$ particles at 51 successive epochs each separated by $\Delta z \approx 0.128$ were saved. From these data, the per-cell shearing forces $\mathcal{R}$ (i.e. $\Phi_{\mathrm{zz}}$ ) were computed from equation $(9)$, along with their column averages $\langle\mathcal{R}\rangle_{\|}$. For comparison, we computed column densities $\Sigma=L \cdot\langle n\rangle_{\|}$, and from these $\mathcal{S}^{[1]}, \mathcal{S}^{[2]}$ evaluated from equations $(16,17)$. In both the 2 -D deflections model and our $C^{0}$ shear model, the convolutions $(9)$ and $(16,17)$ were done by Fast Fourier Transform (FFT). Simple particlein-cell "charge" assignments (see Hockney and Eastwood (1981)) were used here, although the N-body simulation itself is of higher order (see Couchman (1991)). Initial data for the N-body simulation were given by the Zel'dovich prescription with a simple $1 / k$ power spectrum with cut-off (where $R_{L}=128 \mathrm{mpc}$ is the size of the box at the current epoch),

$$
\begin{aligned}
P(k) & =110 \delta^{2}\left(\frac{R_{L}}{2 \pi}\right)^{3} k^{-1} e^{-(k / 0.96)^{16}} \\
\delta & =5 \times 10^{-5} .
\end{aligned}
$$

First note that, indeed, the real and imaginary parts of $\langle\mathcal{R}\rangle_{\|}$are uncorrelated. The correlation coefficient, (e.g. Kalos and Whitlock (1986), section 2.2), of the column means $\left\langle\mathcal{R}^{[1]}\right\rangle_{\|}$, and $\left\langle\mathcal{R}^{[2]}\right\rangle_{\|}$is less than 0.7 percent for each of the 51 epochs.

We now demonstrate that for sufficient softening $(\epsilon \geq 2)$, that the bulk $(\Delta z \sim 1 / 10)$ increments of shear are Gaussian. According to the central limit theorem, a wide class of zero-centered, identically and symmetrically distributed random variables will sum to a normally distributed random variable. This is the case here, where the (locally strongly correlated) individual shearing forces are decidedly non-Gaussian, but in the bulk, a sum of their individual contributions will be. Figure 2 shows the histograms of the real 
and imaginary parts of $\left\langle\Phi_{z z}\right\rangle_{\|}$at the current epoch, timestep 1166 in our simulation. The sample is over the $L^{2}=128^{2}$ pixels of the $\vec{x}_{2}$ plane. These are normalized by $\frac{3}{4}$ to compare with $\mathcal{S}$, equations (16) and (17). The shearing forces in each of the $L^{3}$ cells have cusped distributions. Least squares fits to exponents $\nu$ of the ansatz $p(r)=\mathcal{N}(\nu, \lambda) \exp \left(-\lambda|r|^{\nu}\right)$, where $r$ is either $\left\langle\mathcal{R}^{[1]}\right\rangle_{\|}$or $\left\langle\mathcal{R}^{[2]}\right\rangle_{\|}$, are shown in Figure 3. By eye, this ansatz gives good representations for the distributions of these correlated forces.

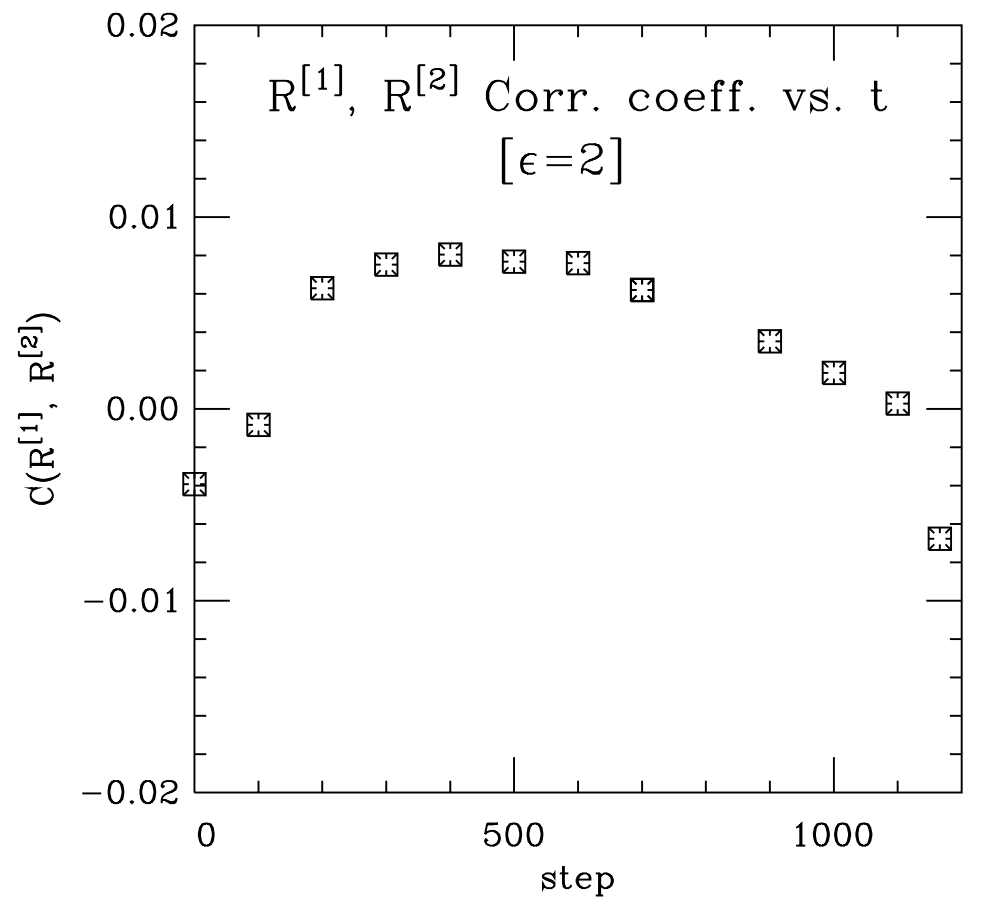

Figure 1: Correlation coefficient between real and imaginary parts of $\left\langle\Phi_{z z}\right\rangle_{\|}$. Samples are taken in the plane normal to the congruence.

Furthermore, such a parameterization permits a crude assessment of the convergence rate to normality of their sums. For $\nu \geq 1 / 2$, we find that a sum of less than 128 such (uncorrelated) random variables is normal with a $\chi^{2}$ confidence level greater than 80 percent. When $\nu<1 / 2$, however, convergence is seriously degraded. This is confirmed by Figures 2 and 5: when $\epsilon \geq 2$, the 128-sum is Gaussian, but for $\epsilon<2$, the resulting sum is too cusped.

\section{$5 \quad$ Integration procedure}

Equation (1), with expression (2) for $\sigma$, is non-linear as expected from Sachs' equations on which it is based. However, since the shearing force is small, an obvious linearization procedure is to use the shear-free function $D_{0}(z)=(2 / 5)\left(1-(1+z)^{-5 / 2}\right)(\Omega=1$ here $)$ in equation (2); see equation $4.47 \mathrm{a}$ in SEF. A procedure for integrating the non-linear case is discussed in section 5.2. 

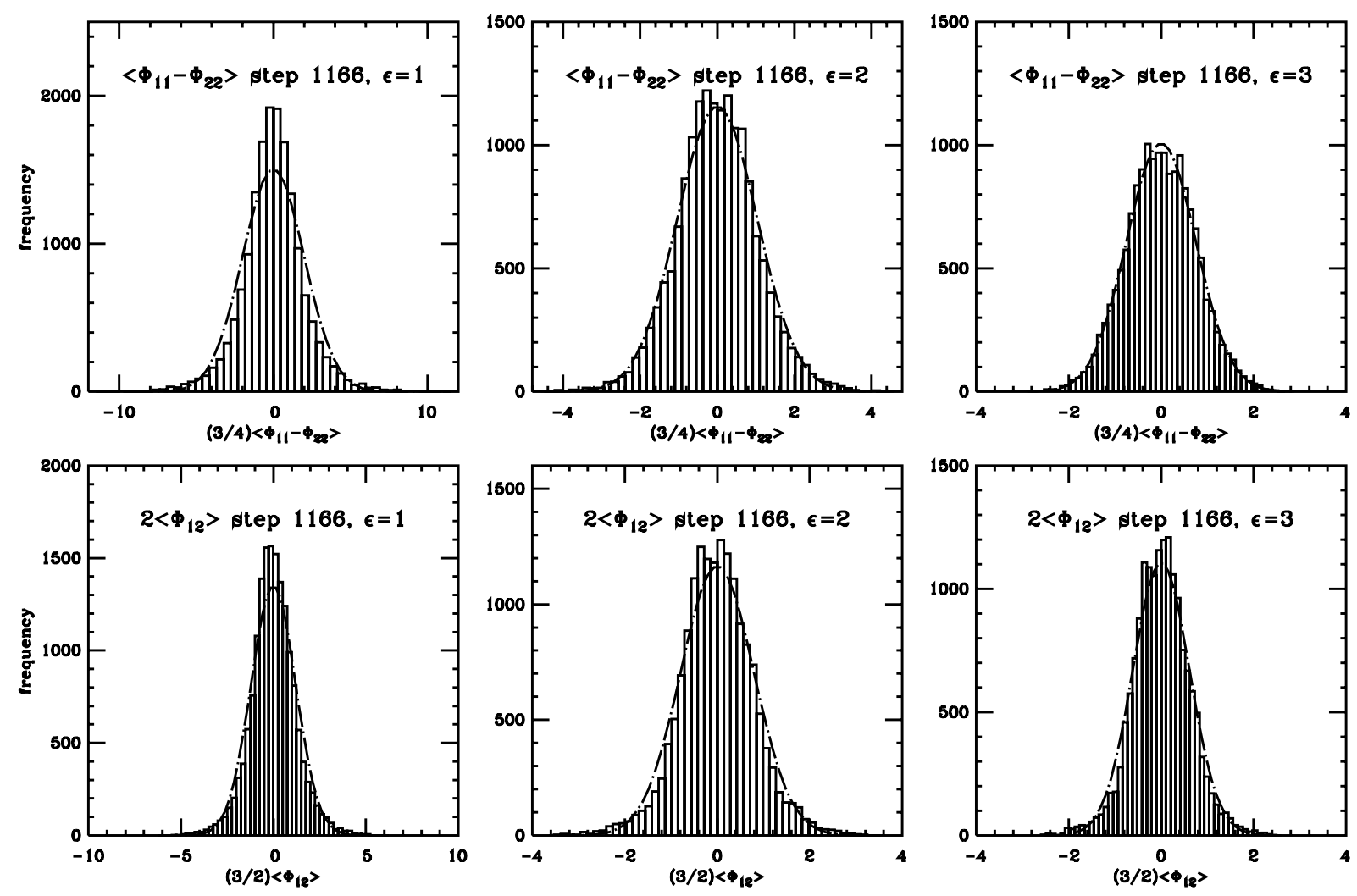

Figure 2: Histograms of real/imaginary parts of $\left\langle\Phi_{\mathrm{zz}}\right\rangle_{\|}$comparing different softenings $\epsilon=1,2,3$. Smooth dashed lines are Gaussian distributions based on the variance. Note that as $\epsilon$ increases, the distributions become more normal.

\subsection{Linearized version}

A second order weak-sense (i.e. it gets all the moments of $\Delta \sigma$ right to $O\left((\Delta z)^{2}\right)$ accuracy) integration procedure for (2) based on our estimate for the increment $\mathcal{R} d z=\mathcal{B}(\xi) d w(\xi)$ is then

$$
\begin{aligned}
\sigma(z)=-\frac{3 \Omega}{8 \pi\left(D_{0}(z)\right)^{2}} & \int_{0}^{z}\left(D_{0}(\xi)\right)^{2}(1+\xi)^{2}(1+\Omega \xi)^{-\frac{1}{2}} \mathcal{B}(\xi) d w(\xi) \\
\approx-\frac{3 \Omega}{16 \pi\left(D_{0}(z)\right)^{2}} & \sum_{i=0}^{\lfloor z / \Delta z\rfloor}\left[\left(D_{0}\left(z_{i}\right)\right)^{2}\left(1+z_{i}\right)^{2}\left(1+\Omega z_{i}\right)^{-\frac{1}{2}} \mathcal{B}\left(z_{i}\right)\right. \\
& \left.+\left(D_{0}\left(z_{i+1}\right)\right)^{2}\left(1+z_{i+1}\right)^{2}\left(1+\Omega z_{i+1}\right)^{-\frac{1}{2}} \mathcal{B}\left(z_{i+1}\right)\right] \Delta w_{i+1}
\end{aligned}
$$

which is a trapezoidal rule. The discrete Brownian increment is $\Delta w_{i}=\sqrt{\Delta z / 2}\left(u_{i}+\right.$ $\left.i v_{i}\right)$, where $u_{i}, v_{i}$ are zero centered, independent, univariant, normally distributed random numbers: $\left\langle u_{i}\right\rangle=\left\langle v_{i}\right\rangle=0,\left\langle u_{i} u_{j}\right\rangle=\left\langle v_{i} v_{j}\right\rangle=\delta_{i j}$, and $\left\langle u_{i} v_{j}\right\rangle=0$ for all $i, j$. One uses the Box-Muller method for these (e.g. Kalos, (1986)). 


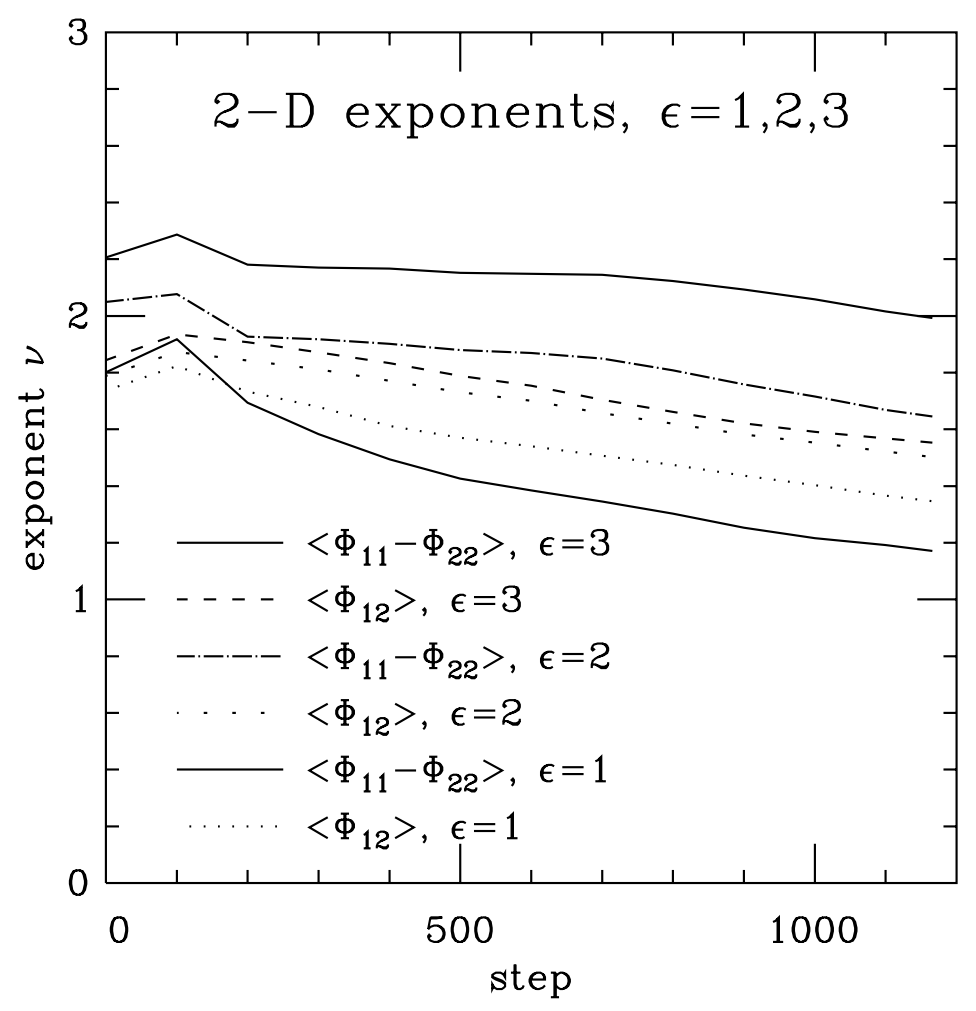

Figure 3: Fits to exponents $(\nu)$ of $r_{i}=\left\langle\mathcal{R}^{[i]}\right\rangle_{\|}$variables vs. number of time steps of N-body simulation: $p\left(r_{i}\right)=\mathcal{N}(\mu, \nu) \exp \left(-\mu\left|r_{i}\right|^{\nu}\right)$. Scale $\mu$, although not shown, varies but is about 2 .

\subsection{Non-linear version}

The non-linear version isn't difficult, either. The best procedure is to use the integral form (2) directly. Since $D$ depends on the complex Brownian process $w$, we need to take care about the interpretation of the integral appearing in the first half of (29).

Namely, since the $d w$ increment is not of bounded variation in interval $d z$ (its autocorrelation yields a $\delta$-function, see (25)), one has to be careful. We chose the Itô rule which assures our result will be a Markov process (e.g. Ikeda and Watanabe (1981)). All that is involved here is to assure that the integrand in (29) is uncorrelated with the increment $d w$. The procedure is simple: we compute an Euler estimate $\sigma_{E}$ for $\sigma$,

$$
\sigma_{E}\left(z_{i+1}\right) \approx\left(\frac{D\left(z_{i}\right)}{D_{E}\left(z_{i+1}\right)}\right)^{2}\left(\sigma\left(z_{i}\right)+\phi_{E} \Delta w_{i+1}\right)
$$

where $\phi(\xi)=-\frac{3 \Omega}{8 \pi}(1+\xi)^{2}(1+\Omega \xi)^{-\frac{1}{2}} \mathcal{B}(\xi), \phi_{E}=\left(\phi\left(z_{i}\right)+\phi\left(z_{i+1}\right)\right) / 2$, and $D_{E}\left(z_{i+1}\right)=$ $D\left(z_{i}\right)+D^{\prime}\left(z_{i}\right) \Delta z$ is the Euler estimate for $D\left(z_{i+1}\right)$. The important thing is that these Euler estimates use only old data (from step $z_{i}$ ) and are thus uncorrelated with $\Delta w_{i+1}$. Now equation (1) may be integrated for one step by trapezoidal rule using Euler estimates for $\left(D, D^{\prime}\right)$, and $\sigma_{E}$. We replace $D_{E}$ in (30) with this new value for $D$ to improve the result for $\sigma$ to finish the step. 

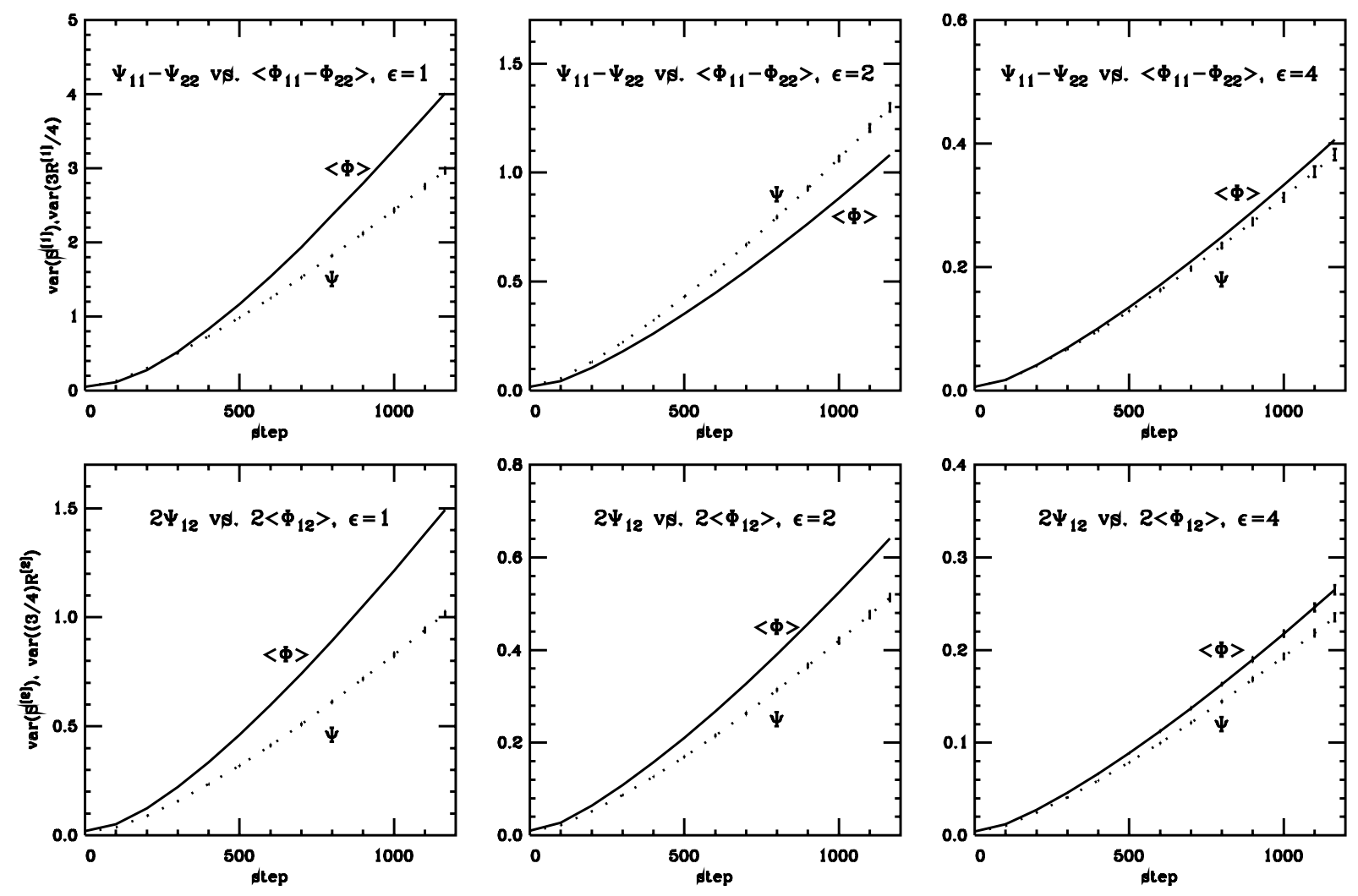

Figure 4: Variances of of $\left\langle\mathcal{R}^{[i]}\right\rangle_{\|}$and $\mathcal{S}^{[i]}$ variables vs. simulation time: variances from the simulations are fit to a power-law behavior in $t=\mathrm{step} / 1166$. The softening parameter values are $\epsilon=1,2,4$. Small error bars show the variation around the mean of choosing $x_{3}=X, Y, Z$ for the congruence direction from the simulation data. To get the coefficients in Table $\mathbf{1}$, multiply the step $=1166$ $(t=1)$ intercept by $(4 / 3)^{2}$.

\section{Results}

In Figure 5, we supposed that the individual contributions of each scatterer have a cusped, but symmetrical distribution. Roughly, these contributions can be fit to distributions of the type $p\left(r_{i}\right)=\mathcal{N} \exp \left(-\mu\left|r_{i}\right|^{\nu}\right)$, where $\nu \in(0.4,2.1)$, and $r_{i}=\mathcal{R}^{[i]}$. The normalization is $\mathcal{N}=\frac{\nu \mu^{1 / \nu}}{2 \Gamma(1 / \nu)}$. A sum of a large number of such contributions would be normally distributed if the distribution satisfies the Lindeberg-Feller condition [13], which they do. Since they are correlated, however, this analysis is not convincing. Simulation provides the only clear demonstration (see Figures 2) that summing $L=128$ of them is Gaussian. From Table 1 , we note that the variances of $\mathcal{R}$ components vary approximately as $t^{1.3} \approx a(z)^{2}$. Were the N-body simulation isotropic, the coefficients of the real and imaginary parts should have the same magnitude. That they don't we ascribe to the fact that the simulation is really only translationly but not rotationally invariant. The representative volume is a cube, not a cylinder. 


\begin{tabular}{|l|c|c|c|c|}
\hline \hline \multicolumn{5}{|c|}{$\mathcal{R}^{[i]}=\gamma_{0} t^{\nu}$ table } \\
\hline $\mathcal{R}$ & $\epsilon=1$ & $\epsilon=2$ & $\epsilon=3$ & $\epsilon=4$ \\
\hline $\operatorname{var}\left(\mathcal{R}^{[1]}\right)$ & $7.22 t^{1.48}$ & $1.90 t^{1.29}$ & $1.01 t^{1.27}$ & $0.68 t^{1.27}$ \\
\hline $\operatorname{var}\left(\mathcal{R}^{[2]}\right)$ & $2.67 t^{1.39}$ & $1.14 t^{1.30}$ & $0.59 t^{1.28}$ & $0.46 t^{1.28}$ \\
\hline $\operatorname{var}(|\mathcal{R}|)$ & - & $3.04 t^{1.30}$ & $1.60 t^{1.28}$ & $1.14 t^{1.28}$ \\
\hline \hline
\end{tabular}

Table 1: LSQ fits to variances of real and imaginary parts of $\mathcal{R}$ variables. If var $\propto a(z)^{2}$, exponent $\nu$ would be 4/3. Here, $t=\operatorname{step} / 1166$ is the fraction of the simulation time.

\subsection{Accuracy of the method}

The linearized version, section 5.1, in fact, seems pretty accurate and is essentially indistinguishable from the non-linear version in Figure 5.2. Indeed, we may use this to get an analytic estimate for the variance of $\sigma$ as follows. Our simplest model (31) for $\mathcal{B}$ is (see exponents in Table 1)

$$
\mathcal{B}(\xi)=\frac{\sqrt{0.128 \gamma_{0}}}{1+\xi}
$$

where $\gamma_{0}=3.04(\epsilon=2$ case $)$ - see Table 1 . Constant 0.128 is the red-shift depth used in the N-body simulation from which the variances of $\left\langle\Phi_{z z}\right\rangle_{\|}$were determined. Squaring equation (7) and taking the expectation value by the rules (25), we get for $\Omega=1$ :

$$
\begin{aligned}
\left\langle|\sigma(z)|^{2}\right\rangle_{\perp} & =\frac{9\left|\gamma_{0}\right|^{2}}{64 \pi^{2}} \frac{1}{(D(z))^{4}} \int_{0}^{z} d \xi(D(\xi))^{4}(1+\xi) \\
& =\frac{18\left|\gamma_{0}\right|^{2}}{320 \pi^{2}(D(z))^{4}} \int_{1}^{X} d y y^{\frac{1}{5}}\left(\frac{y-1}{y}\right)^{4} .
\end{aligned}
$$

where $X=(1+z)^{\frac{5}{2}}$. In the integrand, we've used the explicit form for the shear-free angular diameter distance $D(\xi)=\frac{2}{5}\left(1-(1+\xi)^{-\frac{5}{2}}\right)$ which will also be substituted for $D(z)$ on the right-hand side for the term outside the integral. The integral (32) is trivial, and the 6 term result is then available to integrate equation (1) using an approximation which substitutes $|\sigma|^{2} \rightarrow\left\langle|\sigma|^{2}\right\rangle_{\perp}$. The resulting deterministic (not stochastic) equation is easily computed numerically. We used RKF45 (from Watts and Shampine, available from NETLIB) to do this. From this computation, we can plot the relative error $(\langle D(z)\rangle-$ $\left.D_{a}(z)\right) / D_{a}(z)$ vs. step-size $\Delta z . \quad D_{a}(z)$ is the analytic estimate using the $|\sigma|^{2} \rightarrow\left\langle|\sigma|^{2}\right\rangle_{\perp}$ substitution (32). Additionally, we can get an error estimate for the variance of $D$ by comparing a very small step-size result to the results for various (larger) step-sizes $\Delta z$. Figures 7 show the data for these comparisons. We find that the method is globally at least $O(\Delta z)$ accurate (i.e. locally second order weak accurate: see Kloeden and Grüne (1999)). 


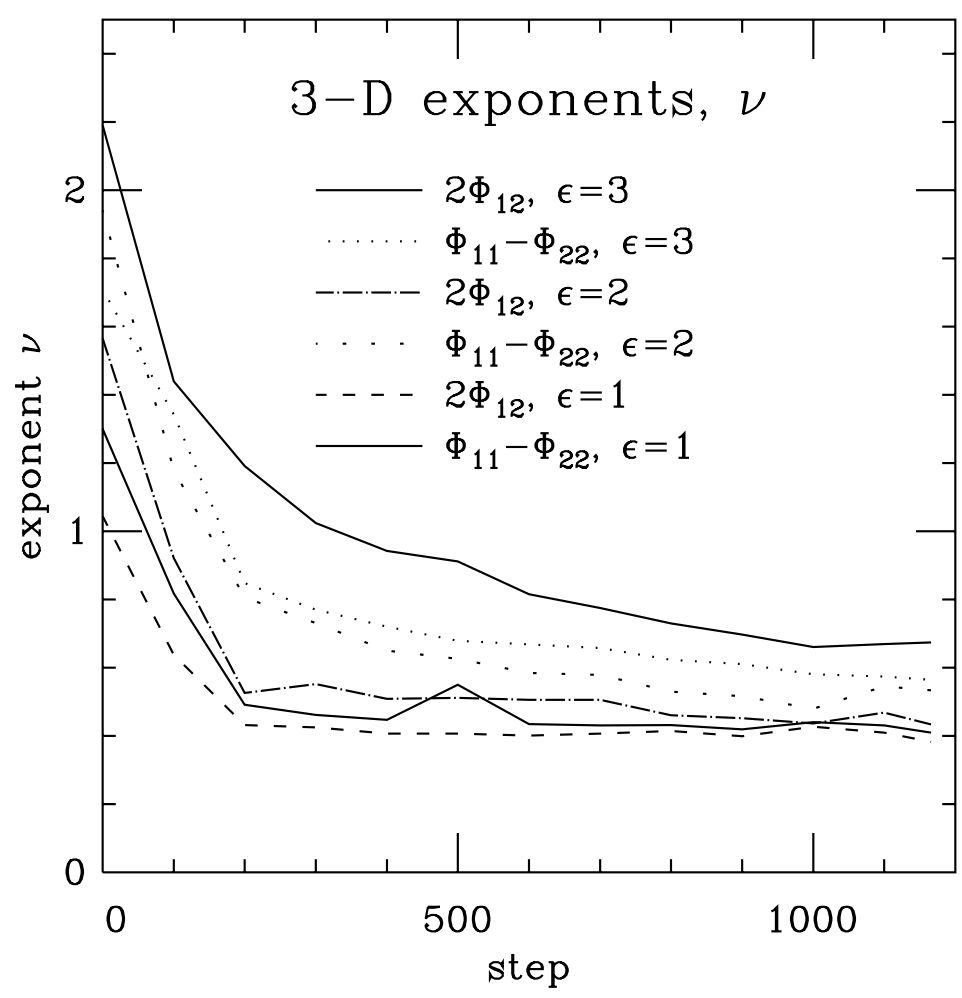

Figure 5: Parameter fits to raw $\Phi_{\mathrm{zz}}$ distribution data. The parametrized model is $p(r)=\mathcal{N} \exp \left(-\mu|r|^{\nu}\right)$, where $\mathcal{N}=\nu \mu^{1 / \nu} /(2 \Gamma(1 / \nu))$ is the normalization, and $r$ is either $\operatorname{Re}\left(\Phi_{\mathrm{zz}}\right)$ or $\operatorname{Im}\left(\Phi_{\mathrm{zz}}\right)$.

\subsection{Some final remarks}

In this final discussion, we comment on the philosophy of the procedure discussed in this paper. In essence, the idea follows the central limit theorem - see, for example, the extended discussion of this theorem in Feller (1974). In its trajectory through what we have called a representative volume, a light ray sees shearing forces due to the inhomogeneous scatterers. If the volume is large enough, these forces add up to a net contribution of (say) $m=\frac{\Delta z}{\delta z}$ scatterers,

$$
\mathcal{Q}=\sum_{i=1}^{m} q_{i}
$$

of $m$ correlated forces $q$. We have argued that if the universe is on-average homogeneous and isotropic, the transverse average is zero, and the distribution is symmetric:

$$
\langle\mathcal{Q}\rangle_{\perp}=0 .
$$

If the sum of $q$ 's is sufficiently large, e.g. $m$ much larger than a cluster correlation length, the central limit theorem would suggest that the distribution for $\mathcal{Q}$ would be Gaussian. All the representation (18) says is that we approximate the above sum of correlated forces 

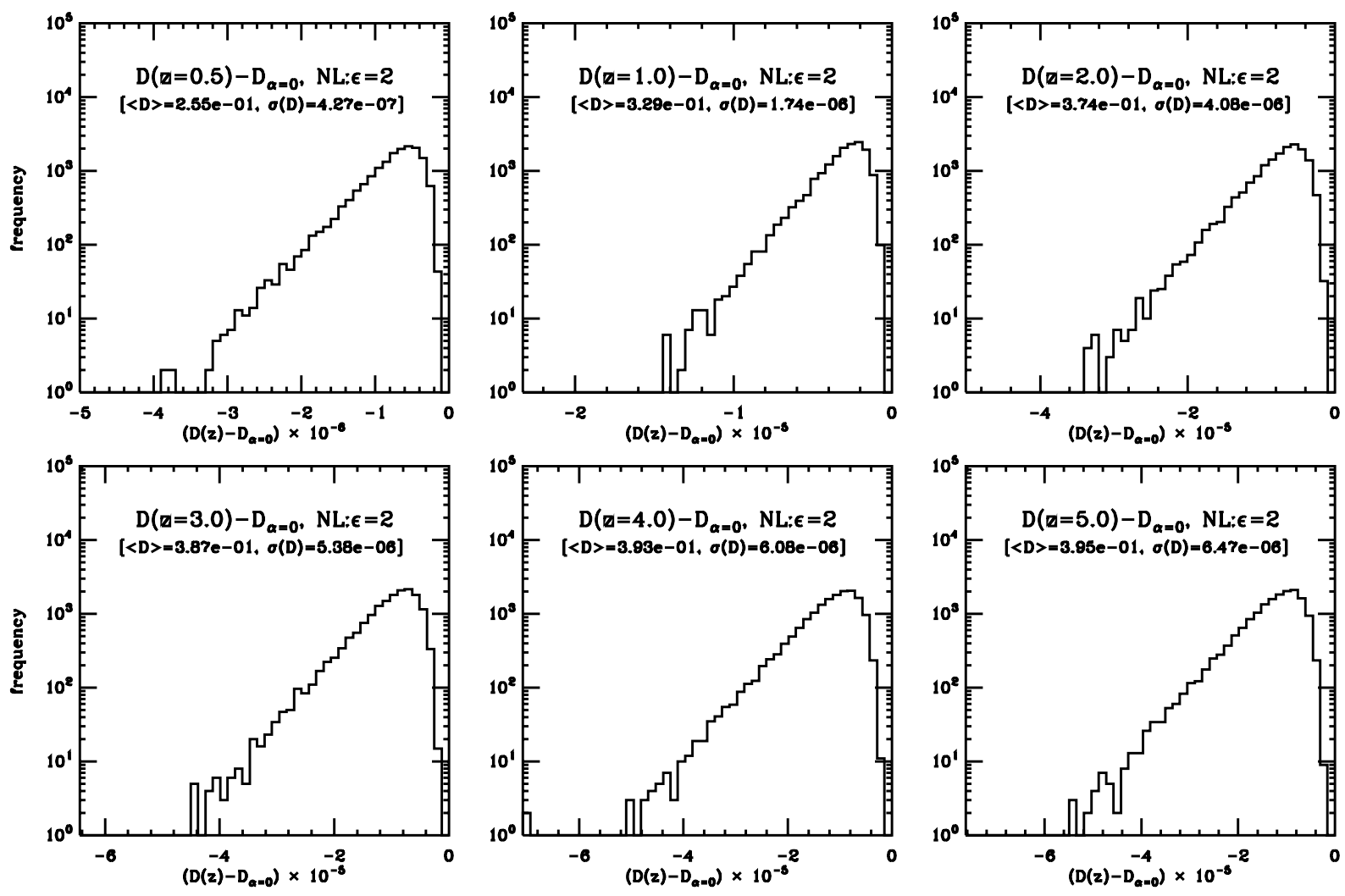

Figure 6: $D(z)$ histograms at $0 \leq z \leq 5$, with $\mathcal{B}=0.62 \cdot a(z)^{1}=0.62 t^{2 / 3}$. These results are from the non-linear integration, section 5.2. Parameter is from Table 1, and is $\sqrt{0.128 \gamma_{0}}$, where $\gamma_{0}$ is from the last row in this table $(\epsilon=$ 2 ). Value 0.128 is the $\Delta z$ stepsize of the $N$-body simulation. Scales for the abscissas are respectively: $10^{-6}$ for the first $(z=1 / 2)$, and $10^{-5}$ for $z=1,2,3,4,5$.

by

$$
\mathcal{Q}=\frac{\mathcal{B}(z)}{\sqrt{\Delta z}} \sum_{i=1}^{m} \delta w_{i}
$$

a sum of complex Gaussian uncorrelated increments $\Delta w=\sum \delta w$. This does not say, however, that the increments $\delta w \propto q$. Indeed, this is a familiar representation of molecular forces - which are locally correlated, and non-normally distributed. In bulk, however, the statistics become more/less normal - as shown in Figure 2. From this, the weighting factor in (33) is proportional to $(\Delta z)^{-1 / 2}$ because the number of scatterers is proportional to $\Delta z$ (see [13], vol. II, sec. VIII.4). $\mathcal{B}(z)$ is a $z$ dependent 2 dimensional diffusion coefficient. The 2-D projection model has all $q=0$ except a central one, whose size must be of order $O(m)$.

Finally, in this paper we have treated only the $\tilde{\alpha}(z)=0$ situation. If this smoothness factor $(0 \leq \tilde{\alpha} \leq 1)$ could be modeled as a function of the column density sums, $\Sigma$ (see Jaroszynski et al. (1990)), we find it will be uncorrelated with the increments of shear. 

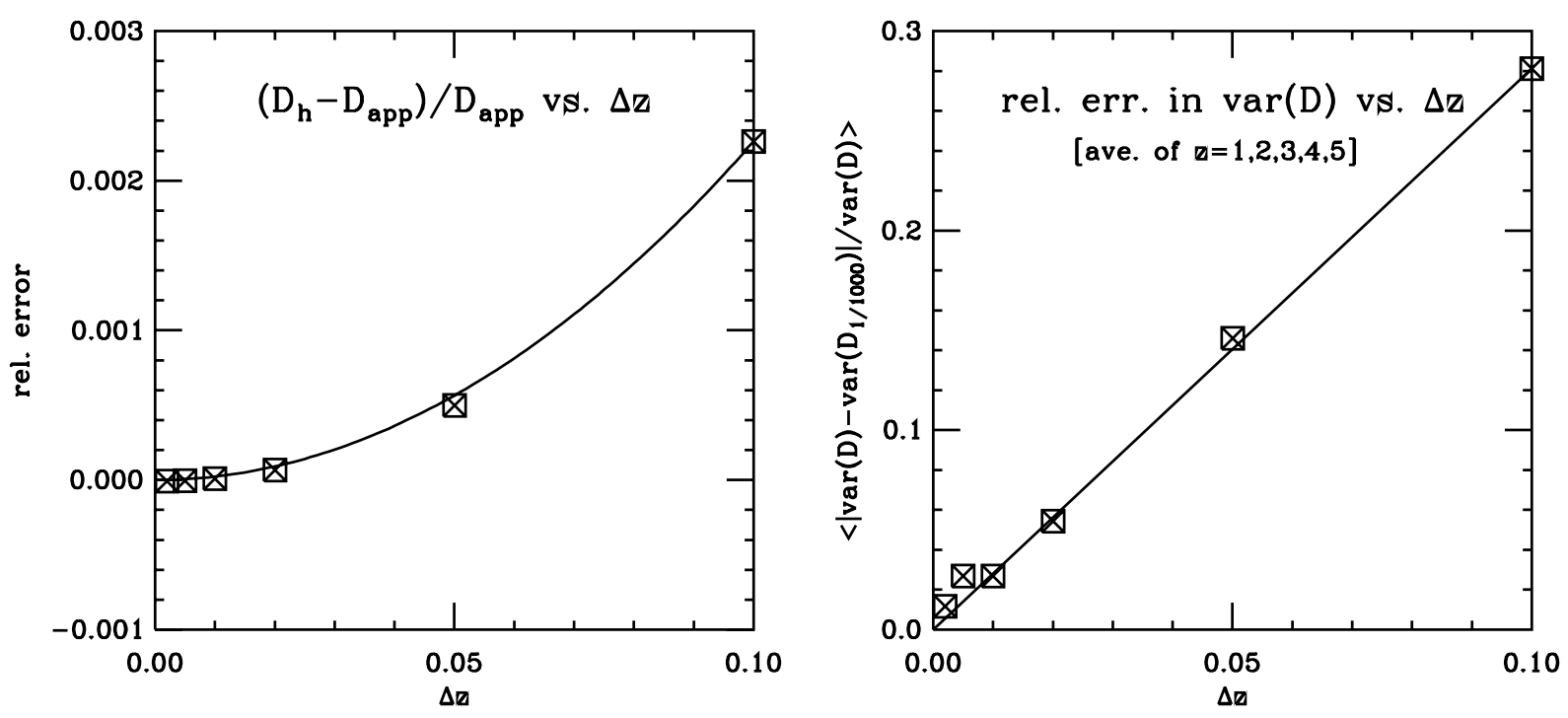

Figure 7: Relative errors in mean and variance vs. step-size $\Delta z$. Approximation $D_{a}$ is from section 6.1, variance errors are relative to $\Delta z=0.001$. In each plot, errors are means of $z=1,2,3,4,5$ cases, and solid lines are quadratic $\left(\alpha \cdot \Delta z^{2}\right)$ and linear $(\beta \cdot \Delta z)$ fits in $\Delta z$, respectively.

Our simulations show that both the column means $\left\langle\Phi_{z z}\right\rangle_{\|}$and the projected 2-D forces $\Psi_{\mathrm{zz}}$ are uncorrelated with $\Sigma$ to less than 1 percent.

\section{$7 \quad$ References}

Blandford, R. \& Narayan, R., 1986, Ap. J., 310, 568.

Couchman, H. M. P., 1991, Ap. J., 368, L23.

Couchman, H. M. P., Barber, A. J., and Thomas, P. A., Oct. 1998, Mon. Not. R. Astron. Soc.

Doob, J. L., Stochastic Processes, 1953, John Wiley, New York.

Dyer, C. C. and Roeder, R. C., 1972, Ap. J., 174, L115.

Dyer, C. C. and Roeder, R. C., 1973, Ap. J., 180, L31.

Feller, William, An Introduction to Probability Theory and Its Applications, 1974, John Wiley, New York.

Hockney, R.W. and Eastwood, J.W., 1981, Computer Simulations Using Particles, McGrawHill, New York.

Ikeda, N. \& Watanabe, S., 1981 Stochastic Differential Equations and Diffusion Processes, Elsevier-North Holland, Kodansha.

Jain, B., Seljak, U., and White, S., pre-print Ray Tracing Simulations of Weak Lensing by Large-Scale Structure, Los Alamos archive astro-ph/9901191, 14 Jan. 1999. 
Jaroszyński, M., Park, C., Paczyński, B., \& Gott, J. R., Ap. J., 1990, 365, 22.

Kalos, M. and Whitlock, P. A., 1986, Monte Carlo Methods, Wiley-Interscience publ., New York.

Kloeden, P. E. \& Grüne, L., 1999, Pathwise Approximation of Random Ordinary Differential Equations, J-W Göthe University Preprint, 26/99.

Kloeden, P. E. \& Platen, E., 1992, Numerical Solution of Stochastic Differential Equations, Springer-Verlag, Berlin.

Milstein, G. N., 1995, Numerical Solutions of Stochastic Differential Equations, Kluwer Academic Press, Dordrecht.

Petersen, W. P., 1998, SIAM J. Numer. Analysis, 35, no. 4, 1439.

Schneider, P., Ehlers, J., \& Falco, E., 1992, Gravitational Lenses, Springer-Verlag, NY.

Schneider, P. \& Weiss, A., 1988a, Ap. J., 327, 526.

ibid., 1988b, Ap. J., 330, 1.

Seitz, S. \& Schneider, P., 1994, Astron. Astrophys., 287, 349. 